\title{
Breeze Gravity Current in a Uniform Flow of Air
}

\author{
M.V. Shokurov", N.Yu. Kraevskaya \\ Marine Hydrophysical Institute, Russian Academy of Sciences, Sevastopol, Russian Federation \\ *e-mail: shokurov.m@gmail.com
}

\begin{abstract}
Breeze circulation is often observed nearby the water basin coasts and usually accompanied by a background synoptic wind. One of the basic dynamically important components of the breeze circulation is gravity current. In the present paper the latter is used as the breeze simplified model. The theory of interaction of gravity current and a uniform synoptic wind are developed. The gravity current in the domain of infinite height in a stationary environment and environment with background flow was considered. To solve this problem the law of conservation of mass and universal property of the Froude number was used, which is true in the steady state.

It is shown that increase of a tail-wind is followed by growth of the gravity current velocity and decrease of its height. The opposite situation is observed at increase of a head wind: the current velocity reduces and its height increases.

Using a Taylor series expansion for small values of the background flow velocity a linear dependence of gravity current velocity on background flow velocity can be obtained. The factor determining the slope of the velocity of gravity current propagation on the background wind speed, which is equal $2 / 3$, is a universal constant.

The theory explains the results of numerical simulation previously obtained by numerous authors. A physical interpretation of dependence of the height and velocity of the gravity current on the background flow velocity is presented.
\end{abstract}

Keywords: gravity current, numerical simulation, the Froude number.

DOI: $10.22449 / 1573-160 X-2017-1-3-10$

(C) 2017, M.V. Shokurov, N.Yu. Kraevskaya

(C) 2017, Physical Oceanography

\section{Introduction}

Gravity, or density currents caused by the density gradient, are wide-spread in nature. They are observed when the water of different density from the surrounding reservoirs or rivers enters the water bodies. In an atmosphere the breeze circulation, mesoscale cold front, squall lines and other phenomena [1] differ in the sources and causes of the density difference and behave as gravity currents. However, the structure and dynamics are universal for the density currents of different spatial and temporal scales in various environments. In the present paper, the gravity current will be considered as a simplified breeze model.

The gravity current structure consists of the following elements: the front, the head of the current, its body (the area which is located behind the head), the Kelvin - Helmholtz vortices and the reverse branch of the current. The head of the current is an area several times higher than the main body of the current. The height of the head is determined by the value of convergence occurring before the front due to a collision of air masses with different physical characteristics. The convergence magnitude is influenced by the difference between the densities of the two colliding masses, background synoptic wind and other factors.

To study the synoptic wind effect on the development and distribution of the gravity currents the laboratory [2], in situ [3], as well as numerical simulations using different models $[1,4-6]$ were carried out. In these studies it is noted that the direction and strength of the synoptic wind significantly affect the structure of 
the atmospheric gravity currents. For example, the most intense breeze fronts, which are determined by the values of vertical velocity or the temperature gradient magnitude, are clearly observed in the head synoptic wind, unlike the hard defined front under the tail wind [3].

The dependence of gravity current propagation velocity on a uniform background synoptic wind velocity was empirically derived in a number of studies [2, 4 - 6]. The following interesting fact was revealed: a gravity current propagation velocity is the sum of the front current velocities and the background wind, multiplied by a coefficient that is unequal to one. This dependence cannot be explained by the principle of relativity of motion and requires a more detailed consideration.

As a rule, the wind profile in the atmosphere is not uniform, it is more complicated. Importance of the background wind shear is shown in the work about thunderstorm squall line maintenance [7]. In [8] the analytical theory describing the interaction of a gravity current and a background flow with a vertical velocity shear is proposed. Subsequently the research on this subject is developed both in analytical works and in the works where the numerical simulation is used [9-12].

The aim of the present paper is to construct a theory to explain the previously obtained dependence of the gravity current velocity and its head height from the background flow velocity.

\section{Problem statement and development of the analytical theory}

Now we are to consider the gravity current with the height $h$, propagating with the velocity $c$ in the unlimited height atmosphere. At the same time, there is a uniform flow, i.e. the synoptic wind, in the atmosphere. Potential gravity current temperature differs from the potential temperature of the environment by the value of $-\Delta \theta$, so that the buoyancy deficit $g^{\prime}$ equals

$$
g^{\prime}=g \frac{\Delta \theta}{\theta_{0}},
$$

where $g$ is the free fall acceleration; $\Delta \theta$ - the difference in potential temperature of the surrounding atmosphere and the gravity current; $\theta_{0}$ is the potential temperature of the surrounding atmosphere.

It will be assumed that that gravity current is created by the source of cold air with a mass flux of $q$. Then, in the steady mode, according to the law of conservation of mass, the following ratio will be performed for the mass flux of the current $q$

$$
q=h_{0} c_{0}=h c,
$$

where $h_{0}$ is the height and $c_{0}$ is the velocity in the current body away from the front; $h$ is the head height; $c$ is the front velocity.

In accordance with the large amount of research of the gravity current, considered in [13], there is a universal relationship between its height and the front velocity, expressed by the dimensionless Froude number Fr:

$$
\mathrm{Fr}=\frac{c}{\sqrt{g^{\prime} h}} .
$$

For the gravity currents the Froude number is a universal constant. 
Case of zero background wind $(\boldsymbol{U}=\mathbf{0})$. The Cartesian coordinate system in the $x z$ plane is considered, where $x$ axis is directed to the right, $z$ axis is directed upwards. Let the gravity current propagate from the source of cold air (cold pool) with a predetermined source mass flux $q$ in a positive direction of $x$ axis. For given constant values of mass flux $q$ and the Froude number Fr the current height $h$ and its velocity $c$ can be obtained from the expressions (1) and (2):

$$
\begin{gathered}
h=\left[\frac{q}{\operatorname{Fr} \sqrt{g^{\prime}}}\right]^{\frac{2}{3}}, \\
c=\left[\operatorname{Fr} \sqrt{g^{\prime} q}\right]^{\frac{2}{3}} .
\end{gathered}
$$

As the expressions (3) and (4) show, in the atmosphere under the fixed predetermined mass flux $q$ and the given cold air source gravity $g$, both the height $h$ and the gravity current velocity $c$ is fully determined by the mass flux $q$ and the Froude number Fr.

Case of the non-zero background wind $(\boldsymbol{U} \neq \mathbf{0})$. This time the gravity current, extending from a source of cold air in the presence of a background flow is considered. It is assumed that the velocity of gravity current $c$ in the fixed reference frame is positive $(c>0)$, and the background flow velocity $U$ can be either positive (tail wind) or negative (headwind).

In the frame of reference associated with the front of the gravity current, the movement of air both inside of the gravity current and in the surrounding atmosphere is stationary. That's why there is the universal relationship between the velocity of the incoming air $(c-U)$ and the gravity current height $h$ :

$$
\mathrm{Fr}=\frac{c-U}{\sqrt{g^{\prime} h}} .
$$

Similar to the previous section of the expressions (1) and (5) the following equations are obtained:

$$
\begin{gathered}
\operatorname{Fr} \sqrt{g^{\prime}} \cdot h^{\frac{3}{2}}+U h-q=0, \\
c^{\frac{3}{2}}-U \sqrt{c}-\operatorname{Fr} \sqrt{g^{\prime} q}=0 .
\end{gathered}
$$

Equations (6) and (7) determine the dependence of the height $h$ and the gravity current velocity $c$ from background wind velocity $U$, as well as from the basic characteristics of the cold air source - its mass flux $q$ and buoyancy deficit $g^{\prime}$.

The obtained expressions are valid not only for atmospheric breeze gravity current, but also for any arbitrary gravity current propagating in the presence of a background flow in the steady state.

Below the graphs of $h(U)$ and $c(U)$ will be constructed, using the values of magnitudes typical for breeze gravity current. The value of the Froude number $\mathrm{Fr}=1$ is chosen (it's the most commonly used value for the gravity currents in an PHYSICAL OCEANOGRAPHY NO. 1 (2017) 
infinitely deep neutral atmosphere [13, 14]). $Q$ mass flux is assumed to be $5000 \mathrm{~m}^{2} / \mathrm{s}$ according to formula (1), since the characteristic values of the breeze circulation are the values $h=1000 \mathrm{~m}$ and $c=5 \mathrm{~m} / \mathrm{s}$. The variation range of the typical background wind velocity values $-20 \mathrm{~m} / \mathrm{s}<U<20 \mathrm{~m} / \mathrm{s}$ is taken. The graphs of the relation between height of the current $h$ (Fig. 1) and its velocity $c$ (Fig. 2) from the background uniform wind the $U$, using the equations (6) and (7) are constructed below. In this case $U<0$ corresponds to headwind, and $U>0-$ to the tail one.

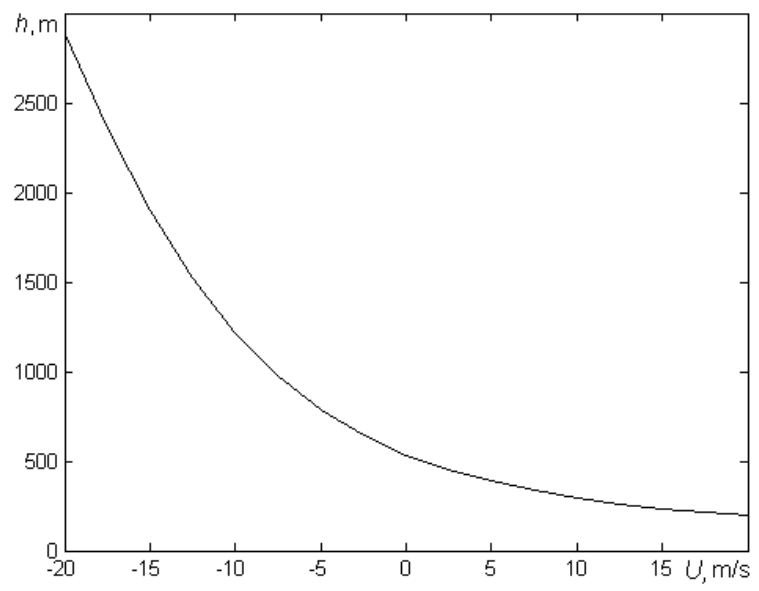

Fig. 1. Dependence of the current height $h$ from the background flow velocity

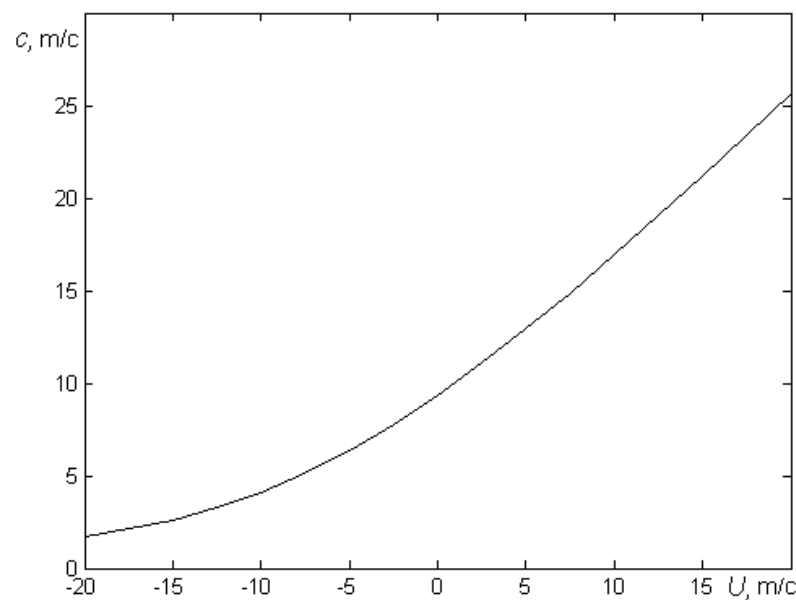

Fig. 2. Dependence of the current velocity $c$ from the background flow velocity $U$

The graphs show that with tail wind velocity increase the gravity current velocity also increases and the height reduces. As follows from the formulas (6) and (7), with an increase of tail background flow velocity $(U \rightarrow \infty)$ the current height will tend to zero $(h \rightarrow 0)$, and the current velocity will be closer to the maximum value, equal to the background wind velocity $(c \rightarrow U)$. 
With the increase of the headwind velocity $(U \rightarrow-\infty)$ the current height will grow $(h \rightarrow \infty)$, and the current velocity will tend to zero $(c \rightarrow 0)$.

Case of the low values of background wind velocity $U$. For low values of $U$ the simpler dependencies of current velocity $c$ and its height $h$, having the linear character, can be obtained (Fig. 1 and 2). To determine them, it's necessary to expand the expression for the velocity and height in a Taylor series in the neighborhood of $U=0$ :

$$
\begin{aligned}
& c(U)=c(0)+\frac{\partial c}{\partial U}(U=0), \\
& \left.h(U)=h(0)+\frac{\partial h}{\partial U}(U=0)\right) U .
\end{aligned}
$$

Substituting these expansions in the formulas (6) and (7), the result is

$$
\begin{array}{r}
c(U)=c(0)+\frac{2}{3} U, \\
h(U)=h(0)-\frac{2}{3} \frac{h_{0}^{1 / 2}}{\sqrt{g^{\prime}} \mathrm{Fr}} U .
\end{array}
$$

It should be noted that the dimensionless ratio of $2 / 3$ before $U$ in the formula (8) does not depend neither on Fr, nor on $q$ and $g^{\prime}$, which testifies its universal character.

\section{Interpretation of the results}

Below the comparison of the obtained results with the results of other works devoted to the study of atmospheric gravity currents is given. Previously the authors had carried out a numerical simulation of the interaction of breeze gravity current with a uniform flow of air of the synoptic origin [6]. Simulation was carried out using a two-dimensional complete hydrodynamic model of the compressed atmosphere. As a result, the dependences of the structure and dynamics of the gravity current on the background wind velocity were studied. Also, it was determined that the current front velocity varied linearly depending on the background wind velocity (Fig. 3).

This dependence can be described by the expression

$$
c(U)=c(0)+\mu U,
$$

where $\mu$ is the dimensionless coefficient, $\mu=0,64$.

As shown in Fig. 4, the current head height decreases with the tail wind velocity increase and increases with the headwind velocity increase.

In [4] in accordance with the numerical simulation results, the linear dependence $c(U)$ for the range of background wind velocity values of $-10 \mathrm{~m} / \mathrm{s}<\mathrm{U}<10 \mathrm{~m} / \mathrm{s}$ with the numerical value of the coefficient $\mu=0.7$ was obtained. Similar results were obtained in the works [ 3 and 5] with the values of the coefficient $\mu$, equal to 0.7 
and 0.74 respectively. As it can be seen from the formula (8), the theoretical value of $\mu=2 / 3$ well corresponds to the dependences obtained in [3-6].

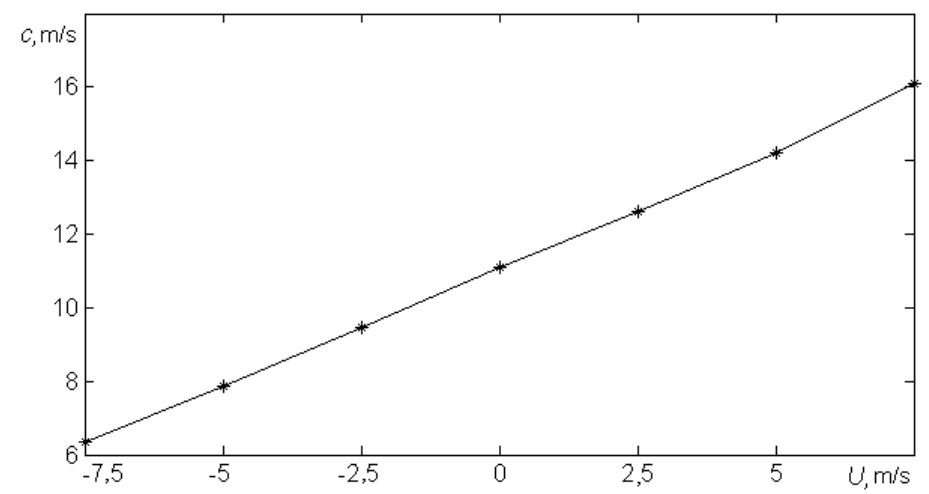

Fig. 3. The dependence of the gravity current front velocity $c(U)$ on the wind velocity $U$. The dots show the results of numerical experiments, the right line - the empirical linear relationship (10)

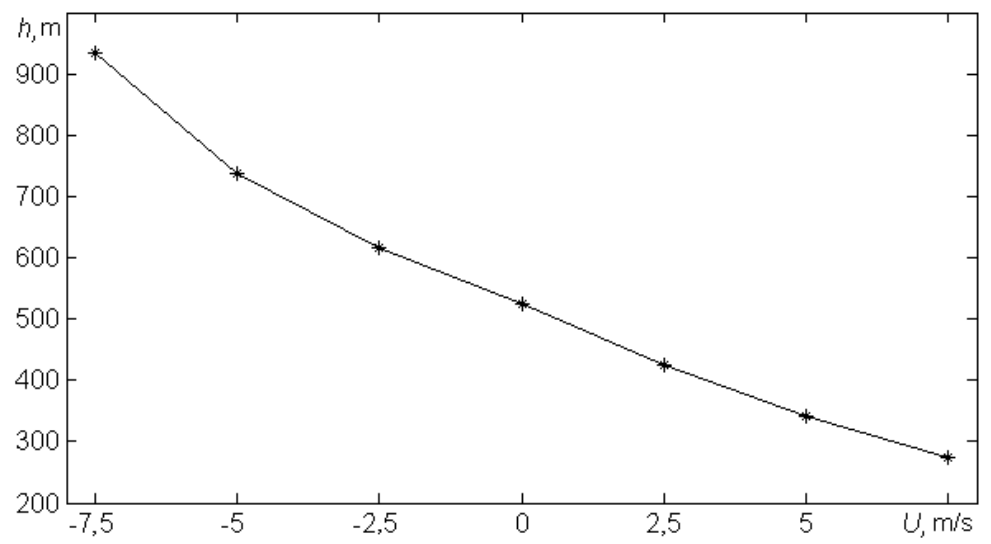

Fig. 4. The dependence of the height of the gravity current head height $h(U)$ on the wind velocity $U$ according to the results of numerical experiments

From the dynamic viewpoint, the height of the current head is defined by a pressure in the stagnation point in the gravity current front. Inside the current the pressure is of hydrostatic characters, gradually decreasing with height. In its turn, the propagation velocity of the current front in the reference frame moving together with the current, depends on the height of the head. Under the head background wind the strong convergence area is formed, resulting in a large value of pressure in the stagnation point. Under the tail wind the convergence before the front is weak, the pressure at the stagnation point is small, and consequently the head of the current cannot reach a great height. This is confirmed by the work [4]. It is noted that there is a range of optimal values of $U$ in the headwind, under which the maximum convergence is observed before the current. Under a strong tail wind the convergence is not observed. 
Earlier in the Section 2 we used the value of the Froude number Fr $=1$ for the theoretical calculations. Theory of interaction of the gravity current with the background wind, developed in the present paper, is valid for unlimited height of the atmosphere. However, numerical and laboratory studies of gravity currents are usually carried out in a limited domain with "solid lid" on the top boundary. In this case, the Froude number will depend on the ratio of the gravity current height to the atmosphere thickness $(h / H)$ and take values in quite a large range of $0.5-1.4$ [12]. For example, in [6] during the simulation the atmosphere height was equal to $5 \mathrm{~km}$. At that, the initial height of the source was $1 \mathrm{~km}$, so that the ratio $h / H$ was 0.2 , and the Froude number took values in the range of $1.1-13$. It follows from what has just been said above that analyzing the results of numerical experiments the special attention should be paid to the choice of the Fr value.

\section{Conclusion}

In this paper a theory of propagation of the stationary gravity current in a uniform flow of air in the atmosphere of unlimited height was developed. The theory is based on the law of conservation of mass for the current mass flux and the Froude number universality. The dependences of the velocity and height of the gravity current head from the background flow are determined. For a certain range of values of the background wind velocity the dependence of the current propagation velocity can be described by a linear function. The obtained value of inclination of dependence of the front velocity, equal to $2 / 3$, is universal and does not depend either on the mass flux, or the Froude number. The results of the analytical theory were compared with the results of numerical experiments. In general, the theory, presented in this paper, can explain the dependence of the height and velocity of gravity current propagation from the background wind.

Acknowledgements. The work was carried out within the Scientific Research Project No. 0827-2015-0001 Climate and No. 0827-2014-0011 Operational Oceanography of Marine Hydrophysical Institute RAS.

\section{REFERENCES}

1. Haase, S.P. and Smith, R.K., 1989. The Numerical Simulation of Atmospheric Gravity Currents. Part I: Neutrally-Stable Environments. Geophys. Astrophys. Fluid Dyn., [e-journal] 46(1-2), pp. 1-33. doi:10.1080/03091928908208902

2. Simpson, J.E. and Britter, R.E., 1980. A Laboratory Model of an Atmospheric Mesofront. Quart. J. Roy. Meteor. Soc., [e-journal] 106(449), pp. 485-500. doi:10.1002/qj.49710644907

3. Atkins, N.T. and Wakimoto, R.M., 1997. Influence of the Synoptic-Scale Flow on Sea Breezes Observed during CaPE. Mon. Wea. Rev., [e-journal] 125(9), pp. 2112-2130. doi:10.1175/1520-0493(1997)125<2112:IOTSSF>2.0.CO;2

4. Thorpe, A.J., Miller, M.J. and Moncrieff, M.W., 1980. Dynamical Models of TwoDimensional Downdraughts. Quart. J. Roy. Meteor. Soc., [e-journal] 106(449), pp. 463-484. doi:10.1002/qj.49710644906

5. Liu, C. and Moncrieff, M.W., 1996. A Numerical Study of the Effects of Ambient Flow and Shear on Density Currents. Mon. Wea. Rev., [e-journal] 124(10), pp. 2282-2303. doi:10.1175/1520-0493(1996)124<2282:ANSOTE >2.0.CO;2

6. Shokurov, M.V. and Germankova, N.Yu., 2015. Vzaimodeystvie Brizovogo Gravitatsionnogo Techeniya s Odnorodnym Potokom Vozdukha [Interaction of the Breeze Gravity Current with the Uniform Flow of Air]. In: Sbornik Materialov X Mezhdunarodnoy Nauchno- 
Prakticheskoy Konferentsii «Fundamental'nye $i$ Prikladnye Issledovaniya v Sovremennom Mire» [Proceedings of the 10th International Scientific-Practical Conference "Fundamental and Applied Studies in the Modern World"]. Saint Petersburg: IIUNTS «Strategiya Budushchego». Vol. 4, pp. 5-16 (in Russian).

7. Rotunno, R., Klemp, J.B. and Weisman, M.L., 1988. A Theory for Strong, Long-Lived Squall Lines. J. Atmos. Sci., [e-journal] 45(3), pp. 463-485. https://doi.org/10.1175/15200469(1988)045<0463:ATFSLL>2.0.CO;2

8. Xu, Q., 1992. Density Currents in Shear Flows-A Two-Fluid Model. J. Atmos. Sci., [ejournal] 49(6), pp. 511-524. doi:10.1175/1520-0469(1992)049<0511:DCISFA>2.0.CO;2

9. Bryan, G.H. and Rotunno, R., 2014. The Optimal State for Gravity Currents in Shear. J. Atmos. Sci., [e-journal] 71(1), pp. 448-468. doi:10.1175/JAS-D-13-0156.1

10. Bryan, G.H. and Rotunno, R., 2014. Gravity Currents in Confined Channels with Environmental Shear. J. Atmos. Sci., [e-journal] 71(3), pp. 1121-1142. doi:10.1175/JAS-D13-0157.1

11. Nasr-Azadani, M.M. and Meiburg, E., 2015. Gravity Current Propagating into Shear. J. Fluid Mech., [e-journal] 778, pp. 552-585. doi:10.1017/jfm.2015.398

12. Benjamin, T.B., 1968. Gravity Currents and Related Phenomena. J. Fluid Mech., [e-journal] 31(2), pp. 209-248. doi:10.1017/S0022112068000133

13. Marino, B.M., Thomas, L.P. and Linden, P.F., 2005. The Front Condition for Gravity Currents. J. Fluid Mech., [e-journal] 536, pp. 49-78. doi:10.1017/S0022112005004933

14. Shokurov, M.V. and Germankova, N.Yu., 2015. Numerical Simulation of Gravity Current Propagation in a Compressible Atmosphere. Physical Oceanography, [e-journal] (4), pp. 5974. doi:10.22449/1573-160X-2015-4-53-65 